\title{
Sjögren-Larsson syndrome: phenotypic heterogeneity in 3 Moroccan siblings
}

\section{Fatima Zahra Elfatoiki, Imane Khrichfa, Hayat Dahbi Skali, Fouzia Hali, Soumia Chiheb}

\author{
Department of Dermatology, Ibn Rochd UHC, 1 quartier des hopitaux 20000, Casablanca, Morocco
}

Corresponding author: Dr. Fatima Zahra Elfatoiki, E-mail: fatiza59@hotmail.fr

\begin{abstract}
Sjögren-Larsson syndrome is a recessively congenital disorder caused by a deficiency of fatty aldehyde dehydrogenase with presenting features of congenital ichthyosis, neurological and ocular involvement. We report three cases of Sjogren-Larsson syndrome in 3 siblings of first-degree consanguineous parents, with history of congenital ichthyosis, psychomotor and speech delays with macular dystrophy. The treatment was symptomatic. Our cases illustrate a rarity and phenotypic heterogeneity of Sjögren-Larsson syndrome. The diagnosis is made by the presence of the classic triad of congenital ichthyosis with pruritus, neurological and ocular involvements and confirmed by enzyme assay, and/or genetic study.
\end{abstract}

Key words: Sjögren-Larsson syndrome; Ichthyosis; Neurological involvement; Macular dystrophy; Consanguinity

\section{INTRODUCTION}

Sjogren-Larsson syndrome is defined as neuro-cutaneous disorder that associated congenital ichthyosis, neurological and ocular features. It is caused by a deficiency of fatty aldehyde dehydrogenase due to mutations in the ALDH3A2 gene. However, phenotypic heterogeneity within a single family affected has been reported [1]. We report cases of Sjogren-Larsson syndrome in 3 siblings of first-degree consanguineous parents, illustrated this clinical heterogeneity.

\section{CASE REPORTS}

\section{Case 1}

A 18-year-old boy, with history at birth of erythematous and thickened skin without true collodion-like membrane. In the first year of live, the skin became dry, itchy, with yellow-brown hyperkeratosis. Skin examination showed generalized ichthyosis and a lichenified appearance of the abdomen, trunk, neck and flexural areas (Fig. 1). On neurological examination, there was severe mental retardation, spasticity and muscular hypertonia of both lower and upper limbs. There were brisk and deep tendon reflexes and bilateral extensor plantar response suggestive of spastic tetraplegia. He was not able to stand or walk on his own and his speech was limited to some meaningful words. On general examination, the patient was wheelchair dependent, with joint contractures and kyphoscoliosis.

\section{Case 2}

14-year-old boy, without history of perinatal complications. Presented with psychomotor and speech delays with itchy skin since a birth. Skin examination showed dry skin with lamellar ichthyosis in flexural areas and lower limbs. On neurological examination, there were deep tendon reflexes and spasticity of both lower limbs with normal tone of upper limbs.

\section{Case 3}

4-year-old girl, with history of erythroderma at birth. Presented with dry and itchy skin with walking disability. On skin examination, there were scaly skin of lower limbs. Neurological examination showed deep tendon

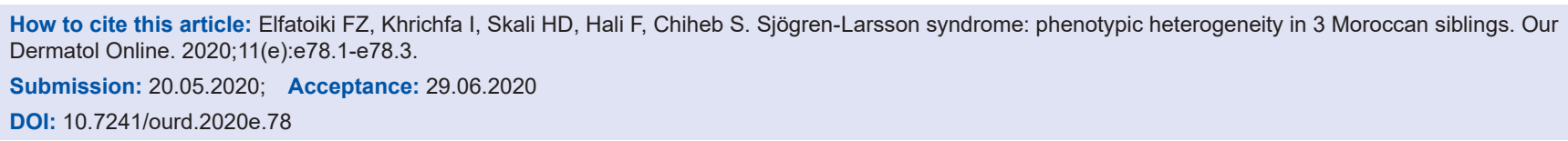


reflexes of lower limbs. The intellectual development was delayed for the age and she was unable to speak full sentences.

\section{Investigations}

Laboratory investigations including routine haematological and biochemical investigations did not show abnormalities. The Electroencephalogram was normal in the three cases. Magnetic resonance imaging of brain in the first case showed bilateral, diffuse and symmetrical T2 hyperintensity in deep white matter of the centrum semiovale suggestive of dysmyelination (Fig. 2).

Histopathology of skin lesions revealed orthokeratotic hyperkeratosis, with irregular acanthosis consistent with ichthyosis. Funduscopic examination revealed the

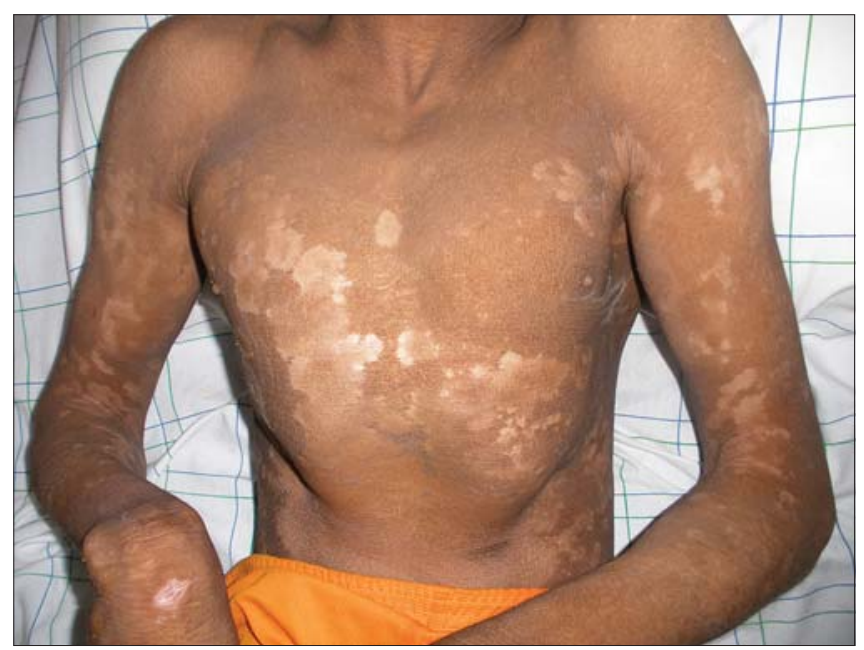

Figure 1: Generalized ichthyosis with a lichenified appearance of the abdomen, trunk and flexural areas.

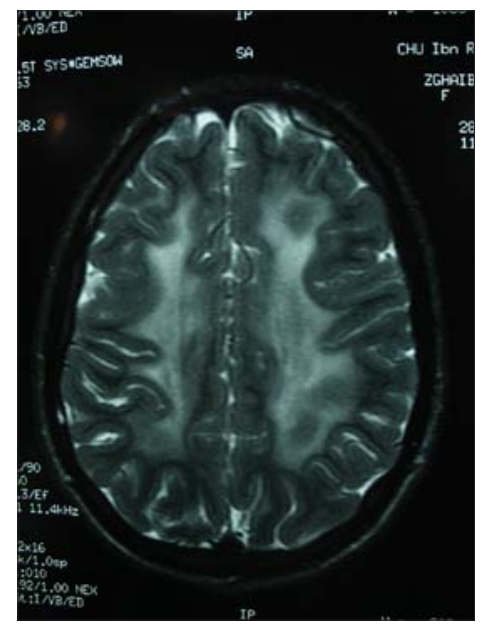

Figure 2: Bilateral, diffuse and symmetrical T2 hyperintensity in deep white matter of the centrum semiovale in magnetic resonance imaging of brain. presence of white and bright spots in macular area in the three cases (Figs. 3a and 3b).

Treatment was provided in the form of physical therapy including language and mobility rehabilitation with family counselling. Cutaneous symptoms were managed with topical moisturising creams and keratolytic agents. No treatment was performed for macular involvement.

\section{DISCUSSION}

Sjogren-Larsson syndrome is an autosomal recessive inborn disorder of lipid metabolism caused by deficient activity of the fatty aldehyde dehydrogenase (FALDH). This defect is due to mutations of ALDH3A2 gene, responsible of FALDH production, an enzyme that catalyzes the oxidation of fatty aldehydes to fatty acids. This defect leads to accumulation of lipid metabolites in the tissues causing various symptoms [2].

The accumulation of fatty acids in the skin destroys the transepidermal water barrier, and leads to ichthyosis and hyperkeratosis. Cutaneous manifestations present at birth as erythroderma or as dry skin and rarely as collodion baby. Ichthyosis appears mainly in the first year of life and mainly involves flexural area, neck, axilla and umbilicus, sparing the face. Palmoplantar keratoderma may be present and pruritus is usually present. Histopathology of the skin biopsy showed hyperkeratosis with keratotic plugging suggestive of ichthyosis $[3,4]$. Our first case had similar cutaneous findings however, the two others had ichthyosis predominantly on lower limbs.

Neurological involvement may manifest in the form of spasticity, dystonia, cognitive or language delays. As was noted in our cases, spasticity is present in the majority of cases and lower limbs are more severely affected than upper limbs. So, most patients become wheelchair dependent during late childhood or early

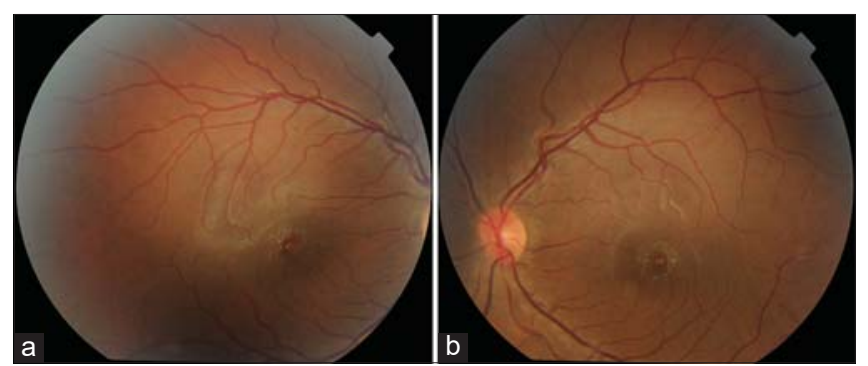

Figure 3: $(a$ and b) Bilateral white and bright spots in macular area in funduscopic examination. 
adolescence as was noted in our first case. Cognitive development is slow associated with mild to moderate mental retardation. Speech and language problems usually are noted due to both motor and cognitive delays $[4,5]$. Our three cases had psychomotor and speech delays.

Brain magnetic resonance imaging showed mild to moderate brain atrophy, and delayed myelination/ dysmyelination appearing as diffuse $\mathrm{T} 2$ hyperintensity in periventricular and subcortical white matter of both cerebral hemispheres, relatively sparing the subcortical U fibres, or in deep white matter centrum semiovale as was observed in our patient [4].

Funduscopy examination is pathognomic of the disease. In the majority of cases, it reveals macular dystrophy with intraretinal crystals appearing as retinal glistening with white dots in the perimacular region of the retina beginning early childhood [2]. These ocular features were noted is our three cases.

The diagnosis of Sjögren-Larsson syndrome is confirmed by measurement of fatty aldehyde dehydrogenase activity or fatty alcohol. The affirmative diagnosis will be obtained by DNA sequencing and identifying mutations in the ALDH3A2 gene. This genetic testing is highly sensitive and can complement or even replace FALDH enzymatic assays [4].

As with most metabolic diseases, there is no curative therapy, and treatment is mainly symptomatic. Physical therapy improved mobility and language acquisition. Few studies showed that 5-lipoxygenase inhibitor, has a potent advantageous effect on pruritus, since it inhibits leukotriene formation. Others may evaluate the efficiency of bezafibrates, carotenoids, and gene therapy in this disorder [1]. In our patients, ichthyosis improved significantly using moisturizing creams, and keratolytic agents associated with physiotherapy.
In conclusion, our cases illustrate a rarity and phenotypic heterogeneity of Sjögren-Larsson syndrome. The diagnosis is made by the presence of the classic triad of congenital ichthyosis with pruritus, neurological and ocular involvements and confirmed by enzyme assay, and/or genetic study.

\section{Consent}

The examination of the patient was conducted according to the Declaration of Helsinki principles.

The authors certify that they have obtained all appropriate patient consent forms. In the form the patient(s) has/have given his/her/their consent for his/her/their images and other clinical information to be reported in the journal. The patients understand that their names and initials will not be published and due efforts will be made to conceal their identity, but anonymity cannot be guaranteed.

\section{REFERENCES}

1. Fuijkschot JT, Theelen T, Seyger MM. Sjögren-Larsson syndrome in clinical practice. J Inherit Metab Dis 2012;35:955-62. 2.

2. Shah V, Rambhia K, Mukhi J, Singh RP. Sjogren-Larsson Syndrome: A Rare Case Report Indian Dermatol Online J. 2018;9:338-40.

3. Rizzo WB. Fatty aldehyde and fatty alcohol metabolism: Review and importance for epidermal structure and function. Biochim Biophys Acta. 2014;1841:377-89.

4. Bindu PS. Sjogren-Larsson Syndrome: Mechanisms and management. Appl Clin Genet. 2020;13:13-24.

5. Willemsen MA, IJlst L, Steijlen PM, Rotteveel JJ, de Jong JG, van Domburg PH, et al. Clinical, biochemical and molecular genetic characteristics of 19 patients with the Sjögren-Larsson syndrome. Brain. 2001;124:1426-37.

Copyright by Fatima Zahra Elfatoiki, et al. This is an open access article distributed under the terms of the Creative Commons Attribution License, which permits unrestricted use, distribution, and reproduction in any medium, provided the original author and source are credited.

Source of Support: Nil, Conflict of Interest: None declared. 